\title{
User Specific Efficiency Parameters for Brain Computer Interface (BCI) based Upper Limb Stroke Rehabilitation System
}

\author{
Azhar Dilshad ${ }^{1}$, AnwaarManzar ${ }^{1}$, Muhammad RizwanTanweer ${ }^{2}$, Tariq Javid ${ }^{1}$, Muhammad AsadUllahKhan ${ }^{3}$ \\ ${ }^{1}$ Faculty of Engineering Sciences and Technology, Hamdard University, Karachi, Pakistan \\ ${ }^{2}$ DHA-Suffa University, Karachi, Pakistan \\ ${ }^{3}$ University of Naples Parthenope, Naples, Italy \\ 1 azhar.dilshad@ hamdard.edu.pk
}

\begin{abstract}
Rehabilitation after stroke through conventional manner is not quite successful due to a number of patient related issues including lack of interest in lengthy exercises, cost of therapy and dependency on healthcare professionals. In addition, around $50 \%$ of stroke survivors worldwide belong to the low and middle income countries that are unable to afford expensive rehabilitation systems. Advancements in Brain Computer Interface (BCI) technology enabling the researchers to design and develop BCI based stroke rehabilitation systems by exploiting neural plasticity. This is achieved via Electroencephalogram (EEG) based computer gaming rehabilitation exercises through Motor Imagery (MI) to achieve successful neural plasticity. However, current research is largely based on expensive bio-signal amplifiers and processing hardware that are beyond the affordability of a large population of stroke patients living in low and middle-income countries. Moreover, the efficiency of BCI based stroke rehabilitation systems that are generally considered as the accuracy of EEG signal classifications is not the only parameter to rate the efficiency. Since the requirements of BCI based rehabilitation therapy are highly subject specific, efficiency of such systems also depends on many user specific features related to cost and performance. This paper describes a research that proposes a number of parameters for cost and efficiency along with their weightage set by the domestic users to determine the overall efficiency of the system. Inputs from different groups of users were obtained that are classified as deserving class, middle class and rich class. Results indicated that the users of different groups are giving different weights to different performance and cost parameters. The overall efficiency requirements are therefore having different meanings for different classes of users.
\end{abstract}

Key words: Brain Computer Interface (BCI), Stroke Rehabilitation, Motor Imagery, BCI performance, BCI efficiency

\section{INTRODUCTION}

There are around 116.4 million stroke cases per year reported worldwide [1] who are living with different levels of disabilities. In many cases, $50 \%$ of survivors found chronically disabled due to severe brain injury [2] and around 30\% of stroke survivors require different types of rehabilitation therapy for upper limb and to restore hand movements [3]. Low and middle-income countries facing $70 \%$ of worldwide strokes cases and in the last four decades; the stroke incidence has increased by more than $100 \%$ in low and middle-income countries. Whereas the stroke occurrence has reduced by $42 \%$ in high-income countries during this period.

Recent advancements in Brain Computer Interface (BCI) provide appropriate techniques to enable the immobilized stroke patients to act and interact with the world through BCI based stroke rehabilitation systems. Motor imagery (MI) based $\mathrm{BCI}$ rehabilitation techniques are considered as an emerging technology to achieve rehabilitation of motor functions [4], [5], [6], [7].

A short review [8] described that neuro-feedback (NF) based training may help to design more individualized task specific NF features, with a patient-specific system that can provide successful rehabilitation. In [9] rehabilitation of the stroke patients is proposed by using a motion tracking device, an EEG feedback system and a virtual reality game. Some more promising $\mathrm{BCI}$ based stroke rehabilitation strategies were reported in the literature [10], [11], [12], [13], [14].

Efficiency of BCI based rehabilitation strategies proposed in the literature is generally considered as the accuracy of classification of brain signals to operate rehabilitation systems. A high level of classification accuracy is provided by using expensive bio signal amplifiers and hardware. Since a large number of stroke patients in the world belong to the low and middle-income countries, the meanings of efficiency of the BCI based rehabilitation systems is largely dependent on their affordability even with compromise on systems accuracy. In addition, other features like interest and curiosity, comfort and usability, number of electrodes, battery capacity and computation requirements may also be compromised to get a "cost efficient" system to those who cannot afford expensive systems. Since the requirements of BCI based rehabilitation therapy are highly subject specific, efficiency of such systems also depends on many user specific features related to cost and performance. However, it is necessary to determine what type of features and factors have more importance by the users in low and middle counters to select the level of features to be provided. 
Azhar Dilshad et al., International Journal of Emerging Trends in Engineering Research, 9(4), April 2021, 524 - 528

This paper describes a research that proposes a number of parameters for efficiency and cost along with their weightage set by the domestic users to determine the overall efficiency of the system. To get the insight on subject matter from all segments of the population, inputs from different groups of users were obtained that are classified as deserving class, middle class and rich class. Results indicated that the users of different groups are giving different weights to different performance and cost parameters and the overall efficiency requirements are therefore having different meanings for different classes of users.

\section{RESEARCH METHODOLOGY}

This research was conducted to support the design and development of a BCI based stroke rehabilitation system as depicted in figure-1 [15], [16]. The proposed system will consist of a bio-signal amplifier, data processing module, VR and Game controlling module along with a feedback module. The aim is to provide all components as per need of the domestic users. The efficiency of the proposed system will depend upon many user centric factors including cost, ease of use, wear-ability, comfort, interesting gaming themes etc. In the subsequent section, overall efficiency based on these factors is discussed.

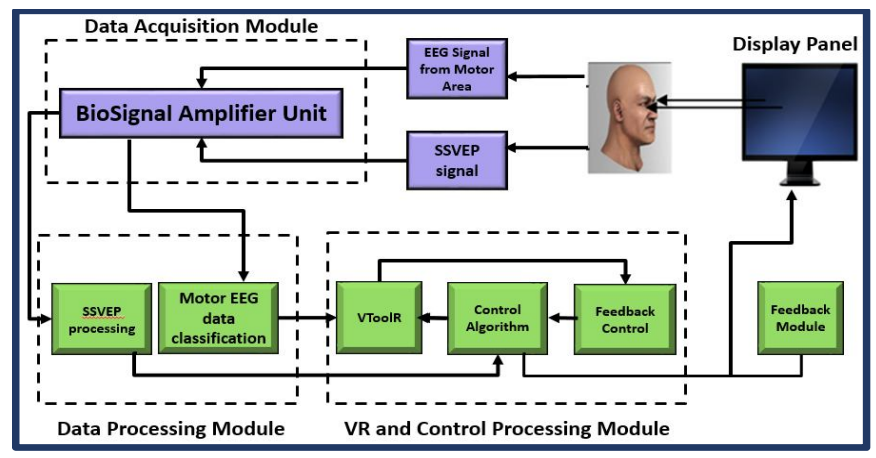

Figure-1. Proposed BCI-Rehab System

Efficiency of the BCI based neuro-rehabilitation system is dependent on various parameters related to performance and cost.Users in different regions of the world may have different levels of choices. As per our best knowledge, no comprehensive formulation is available to compute efficiency of the BCI based rehabilitation system as per user's demand of performance factors and their affordability (cost). In this section, we have proposed that efficiency of such systemscan be formulated through statistical methods.

\subsection{PROPOSED EFFICIENCY PARAMETERS}

As the efficiency of engineering systems is the ratio of their performance $(\mathrm{P})$ to their cost $(\mathrm{C})$.

$$
\text { Efficiency }\left(E_{f}\right)=\frac{\text { PERFORMANCE }(\mathrm{P})}{\operatorname{COST}(\mathrm{C})} \times 100 \%
$$

Assuming that, if for the said application, performance is governed by;

where,
$\mathrm{A}=$ User-interest in gaming themes
$\mathrm{B}=$ Ease of use of VR tool
$\mathrm{D}=$ Comfort in terms of wear-ability
$\mathrm{F}=$ Control on game in terms of accuracy
$\mathrm{G}=$ Independence $/$ Training less
$\mathrm{H}=$ Feedback other than visual
$\mathrm{I}=$ Diversified gaming themes
$\mathrm{J}=$ different forms of feedbacks

For the said application, cost can be governed by

where,

$$
C=f(J c,, K, L, M, N, O)
$$

$$
\begin{aligned}
& \mathrm{J}_{\mathrm{c}}=\text { Number of Channels/electrodes } \\
& \mathrm{K}=\text { Console-features } \\
& \mathrm{L}=\text { Battery-backup } \\
& \mathrm{M}=\text { Data acquisition system } \\
& \mathrm{N}=\text { Warranty of the system } \\
& \mathrm{O}=\text { Computing requirements }
\end{aligned}
$$

Then, from (i) the efficiency Eff will be,

$$
E f=\frac{\mathrm{P}}{\mathrm{C}}=\frac{f(A, B, D, F, G, H, I, J)}{f(J c, K, L, M, N, O)} \times 100 \% \ldots \ldots \text { (ii) }
$$

The function $f$ for both performance and cost is proposed to be determined statistically from the inputs given by the domestic users which can be a general representation for the users living in south Asian countries.

\subsection{STUDY DESIGN}

To obtain the users' weightage for each of the performance and cost parameters, two separate questionnaires were prepared that described each parameter to the participants and asked them to enter the values to rate their requirements for each factor related to performance and cost. In order to get equal feedback from all segments of the society, 50 participants were selected from eachof the three classes, the deserving class, the middle class and the rich class. Each participant was then asked to fill the values against each performance parameters, viz., userinterest in gaming themes, ease of use of VR tool, comfort in terms of wear-ability, control on game in terms of accuracy, independence / Training less, feedback other than visual and Diversified gaming themesfrom 10 (most desirable) to 2 (least desirable). Similarly, each participant entered the values for cost parameters, number of channels/electrodes, consolefeatures, battery-backup, data acquisition system, warranty of the system, computing requirements from 10 (highly affordable) to 1 (least affordable).

\section{RESULTS AND DISCUSSION}

The data obtained from the participants were recorded in two separate tables for performance and cost parameters. The average and standard deviation for each parameter were computed for each group of participants (deserving class, middle class and rich class) as presented in table 1 and table 2 . 
Azhar Dilshad et al., International Journal of Emerging Trends in Engineering Research, 9(4), April 2021, 524 - 528

Table 1: Average values with Standard Deviation for performance parameters entered by the participants.

\begin{tabular}{|c|c|c|c|c|c|c|c|c|c|}
\hline \multicolumn{10}{|c|}{ Performance Parameters (Least desired (1), Most desired (10)) } \\
\hline & A & B & $\mathrm{D}$ & $\mathrm{F}$ & G & $\mathrm{H}$ & I & $\mathrm{J}$ & $\begin{array}{l}\text { Participants' } \\
\text { Group }\end{array}$ \\
\hline Average & 2.64 & 4.26 & 2.86 & 2.26 & 4.36 & 3.38 & 4.04 & 4.44 & \multirow{2}{*}{$\begin{array}{l}\text { Deserving } \\
\text { Class }\end{array}$} \\
\hline $\begin{array}{c}\text { Standard } \\
\text { Deviation }\end{array}$ & 1.34 & 1.23 & 0.86 & 1.12 & 1.24 & 1.19 & 0.78 & 1.05 & \\
\hline Average & 5.04 & 5.1 & 5.1 & 5.36 & 5.54 & 5.12 & 5.08 & 5.28 & \multirow{2}{*}{ Middle Class } \\
\hline $\begin{array}{c}\text { Standard } \\
\text { Deviation }\end{array}$ & 1.44 & 1.5 & 1.27 & 1.26 & 1.36 & 1.38 & 1.26 & 1.36 & \\
\hline Average & 7 & 7.04 & 7.3 & 6.64 & 7.52 & 7.18 & 6.98 & 6.98 & \multirow{2}{*}{ Rich Class } \\
\hline $\begin{array}{c}\text { Standard } \\
\text { Deviation }\end{array}$ & 1.78 & 2.05 & 2.07 & 2.28 & 1.79 & 2.02 & 2.06 & 1.86 & \\
\hline
\end{tabular}

Table 2: Average values with Standard Deviation for cost parameters entered by the participants.

\begin{tabular}{|c|c|c|c|c|c|c|c|}
\hline \multicolumn{7}{|c|}{ Cost Rating (highly affordable = 10 to least affordable = 2 } \\
\hline & $\mathrm{Jc}$ & $\mathrm{K}$ & $\mathrm{L}$ & $\mathrm{M}$ & $\mathrm{N}$ & $\mathrm{O}$ & \\
\hline Average & 3.12 & 2.98 & 3.28 & 3.42 & 2.82 & 2.98 & \multirow{2}{*}{$\begin{array}{c}\text { Deserving } \\
\text { Class }\end{array}$} \\
\hline $\begin{array}{c}\text { Standard } \\
\text { Deviation }\end{array}$ & 1.36 & 1.3 & 1.41 & 1.51 & 1.57 & 1.33 & \multirow{2}{*}{ Middle Class } \\
\hline Average & 4.28 & 4.8 & 4.76 & 4.3 & 4.34 & 4.64 & \multirow{2}{*}{ Rich Class } \\
\hline $\begin{array}{c}\text { Standard } \\
\text { Deviation }\end{array}$ & 1.69 & 1.67 & 1.73 & 1.67 & 1.69 & 1.66 & 7.24 \\
\hline Average & 7.44 & 7.16 & 7.6 & 7.62 & 7.4 & 1.84 & \\
\hline $\begin{array}{c}\text { Standard } \\
\text { Deviation }\end{array}$ & 1.63 & 1.67 & 1.84 & 1.75 & 1.86 & &
\end{tabular}

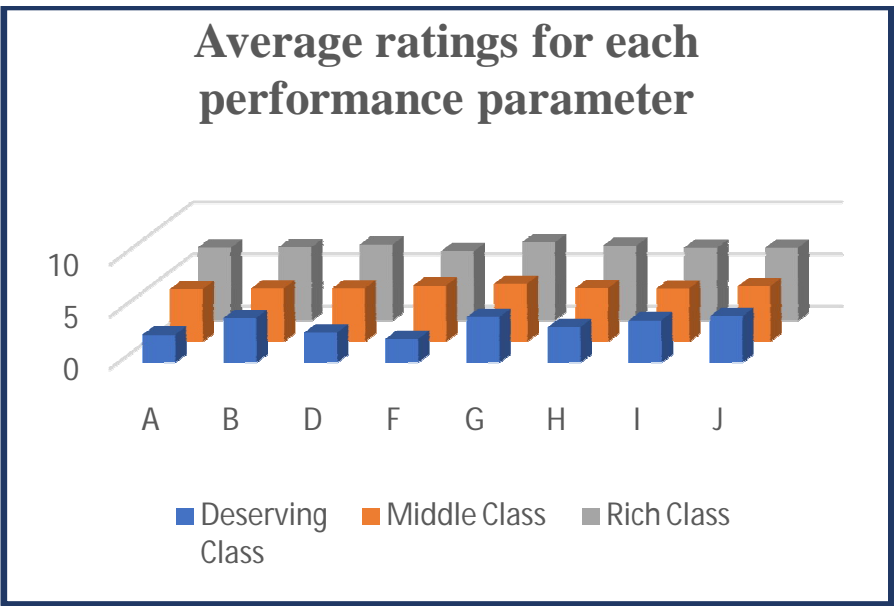

Figure 2. Average ratings for each performance parameter (Least desired $=1$, Most desired $=10$ )

\section{Average ratings for each cost parameter}

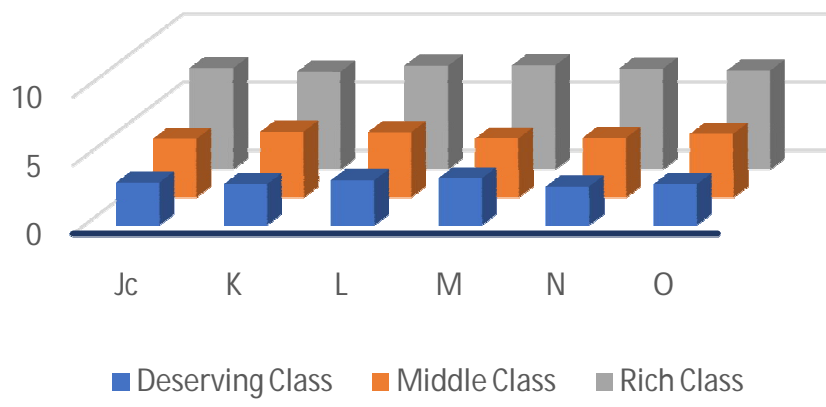

Figure 3. Average ratings for cost parameters (highly affordable $=10$ to least affordable $=2$ )

The results clearly indicate that the choices for desired performance parameters are largely different for each group of participants. Deserving class is too conservative to rate the desired performance parameters as they entered in relatively 
low ranges. This can be considered as their acceptability of rehabilitation systems with relatively low performance. The middle class showed careful attitude while selecting the desired performance and entered almost all performance parameters with mid values which indicated their acceptability of a system with average performance. Rich class showed a surprising behavior while entering desired performance values as they selected up to around $70 \%$ performance in all parameters. This shows that they are comfortable with above average to good performance of the system while excellent performance of the system is either not desired or not expected. However, the selection of cost parameters by each group is not surprising as deserving class have selected up to around $30 \%$, middle class up to around $45 \%$ and rich class selected up to around $75 \%$.

The results also indicate that from prospective of the users, performance of BCI based stroke rehabilitation system will not depend on a few parameters while largely depends on the affordability of the user.

Once we have the users selected values for performance and cost, function $f$ for both could be obtained in the form of a polynomial tree, for example.

$$
E f=\frac{\mathrm{P}}{\mathrm{C}}=\frac{(\alpha+k A+l B+m D+\cdots)}{(\beta+q J+r K+s M-t N+\cdots)} \times 100 \ldots \ldots
$$

Where $\alpha$ and $\beta$ are bias values; $k, l, m, q, r, s, t$ are the corresponding weights to be determined.

To formulate such an expression to express the efficiency of the BCI rehabilitation system based on user selected factors, Linear Regression by using ' $\mathrm{R}$ ' software is proposed. Following expressions for overall efficiency of BCI based rehabilitation system in terms of performance $P$ and cost $C$ are obtained based on the data from all 150 participants acquired from this study.

$$
\begin{aligned}
& P=\alpha+(13.443 \mathrm{~A}+11.132 \mathrm{~B}+13.779 \mathrm{D}+12.590 \mathrm{~F}+14.076 \\
& \mathrm{G}+13.138 \mathrm{H}+13.845 \mathrm{I}+13.4153 \mathrm{~J}) / 8 \ldots \ldots \text { (iv) } \\
& C=\beta+(11.975 \mathrm{Jc}+12.324 \mathrm{~K}+12.002 \mathrm{~L}+11.964 \mathrm{M}+ \\
& 12.3475 \mathrm{~N}+12.427 \mathrm{O}) / 6 \ldots \ldots \text { (v) }
\end{aligned}
$$

Overall efficacy determined through above expressions for each group based on average performance and cost parameters is summarized in table 3 and illustrated in figure 4.

Table 3. Overall efficiency based on average parameters

\begin{tabular}{|c|c|c|c|}
\hline & $\begin{array}{c}\text { Performance } \\
(P) \text { weight }\end{array}$ & $\begin{array}{c}\text { Cost }(C) \\
\text { weight }\end{array}$ & $\begin{array}{c}\text { Overall } \\
\text { Efficiency (\%) }\end{array}$ \\
\hline $\begin{array}{c}\text { Deserving } \\
\text { Class }\end{array}$ & 40.51 & 45.69 & 88.66 \\
\hline $\begin{array}{c}\text { Middle } \\
\text { Class }\end{array}$ & 62.59 & 63.02 & 99.31 \\
\hline $\begin{array}{c}\text { Rich } \\
\text { Class }\end{array}$ & 87.40 & 98.15 & 89.04 \\
\hline
\end{tabular}

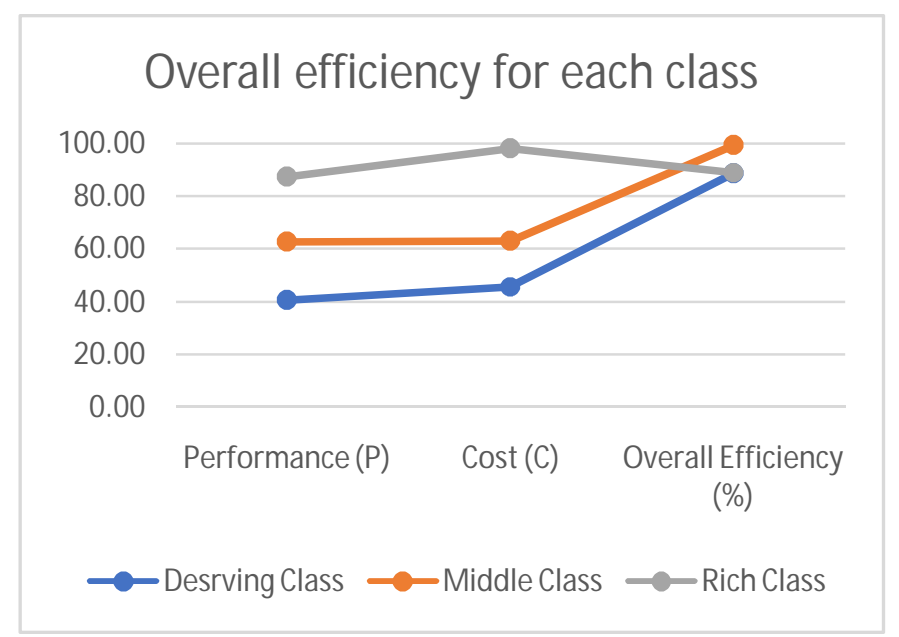

Figure 4. Overall efficiency based on average parameters

The figures in table 3 suggest that from the point of view of deserving class the overall efficiency of the system will be $88.66 \%$ by accepting average performance at relatively less cost. For the middle class the system will be highly efficient (99.3\%) with moderate performance in average cost while for the rich class the system will be $89 \%$ efficient with very good performance at almost maximum cost. However, this approximation is based on the values of a particular region which may vary if data collected in another region of the world.

\section{CONCLUSION}

Research on design and development of Brain Computer Interface (BCI) based upper limb stroke rehabilitation system is increasingly reported in recent literature. Proposed systems are mainly considering the accuracy of EEG signal classification to rate the efficiency. High accuracy is obtained via expensive biosignal amplifiers and large number of EEG electrodes used in the research. Other features like interest in rehabilitation gaming, comfort, ease of use are also contributing in the overall performance. A large number of stroke survivors who require rehabilitation therapy are belong to low and middle-income countries for them expensive BCI systems are beyond to afford. A number of parameters are proposed that need to be considered for a BCI rehabilitation system to rate the overall efficiency in terms of both performance and cost. An expression to formulate the the overall efficiency of a BCI rehabilitation system based on the weights for performance and cost parameters given by 150 domestic participants was obtained through statistical method. Results suggesting that from the point of view of domestic users in different social classes, meaning of systems overall efficiency is quite different as they have different choices of performance and cost parameters. The study was based on the domestic users and the findings may vary if data will be collected in another region. Further research may target the improvements in the expression of efficiency formulated in this study. 


\section{REFERENCES} 2019.
'GBD 2016 Stroke Collaborators', “Global, regional, and national burden of stroke, 1990-2016: a systematic analysis for the Global Burden of Disease Study 2016", Lancet Neurol; Volume 18: 439-58,

Eric S. Donkor, "Stroke in the 21st Century: A Snapshot of the Burden, Epidemiology, and Quality of Life", Hindawi, Stroke Research and Treatment, Volume 2018, 2018.

Cuntai Guan, "Brain-Computer Interface for Stroke Rehabilitation with Clinical Studies", International IEEE Winter Workshop on Brain-Computer Interface (BCI), 2013.

Luz Maria Alonso-Valerdi, Ricardo Antonio SalidoRuiz and Ricardo A. Ramirez-Mendoza, Motor imagery based brain-computer interfaces: An emerging technology to rehabilitate motor deficits, Neuropsychologia, Elsevier, 2015

AnushaVenkatakrishnan, Gerard E. Francisco and J. L. Contreras-Vidal, "Applications of Brain-Machine Interface Systems in Stroke Recovery and Rehabilitation", Curr Phys Med Rehabil Rep, 2:93105, 2014.

Lin Yao, JianjunMeng, Dingguo Zhang, Xinjun Sheng, and Xiangyang Zhu, "Combining Motor Imagery With Selective Sensation Toward a HybridModality BCI", IEEE Transactions on Biomedical Engineering, VOL. 61, NO. 4, August 2014.

Tsung-Pen Chou, Wan-Ru Wang and Tian Sheuan Chang, "Low Complexity Real Time BCI for Stroke Rehabilitation”, 2015 IEEE International Conference on Digital Signal Processing (DSP), Singapore, 2015. [C. Jeunet, F. Lotte, J-M. Batail, P. Philip, J-A. MicoulaudFranchi, "Using recent BCI literature to deepen our understanding of clinical neurofeedback: A short review", Neuroscience, 2018.

B.-S. Lin et al, "Novel Upper-Limb Rehabilitation System Based on Attention Technology for PostStroke Patients: A Preliminary Study", IEEE Access, Volume 6, 2018.

Penaloza et al, "Android Feedback-Based Training Modulates Sensorimotor Rhythms", IEEE Transactions on Neural Systems and Rehabilitation Engineering, VOL. 26, NO. 3, 2018.

David Achanccaray et al, "A virtual reality and brain computer interface system for upper limb rehabilitation of post stroke patients", IEEE International Conference on Fuzzy Systems (FUZZIEEE), 2017.

Monge-Pereira. E, Ibañez-Pereda J, Alguacil-Diego. IM, Serrano JI, Spottorno-Rubio. MP, Molina-Rueda. F, "Use of Electroencephalography Brain Computer Interface systems as a rehabilitative approach for upper limb function after a stroke. A systematic review", $P M \& R$, April, 2017.
[13] Ryan Spicer, Julia Anglin, David M. Krum, and Sook-Lei Liew, "REINVENT: A Low-Cost, Virtual Reality Brain-Computer Interface for Severe Stroke Upper Limb Motor Recovery”, 2017 IEEE Virtual Reality (VR), Los Angeles, CA, USA, March 18-22, 2017.

[14] Colin M. McCrimmon et al, "Performance Assessment of a Custom, Portable, and Low-Cost Brain-Computer Interface Platform", IEEE Transactions on Biomedical Engineering, Volume: 64, Issue: 10, 2017.

[15] Azhar Dilshad, Dr. Vali Uddin, "Development of Efficient Brain Computer Interface (BCI) System for Stroke Rehabilitation", IEEE, INMIC 2014.

[16] Azhar Dilshad, Dr. Vali Uddin, Abdul MujeebMemon, UzmaNaz, Sadia Parveen, Dr. Tariq Javid, "Towards Indigenous Development of a General Purpose Steady State Visual Evoked Potential (SSVEP) based Embedded Control Panel for Brain Computer Interface (BCI) Applications",Asian Journal of Engineering, Sciences and Technology, 2016 